\title{
Assessing Correlations Between Lingual Movements and Formants
}

Christopher Dromey

Brigham Young University, dromey@byu.edu

Gwi-Ok Jang

Brigham Young University

Kristi Hollis

Brigham Young University

Follow this and additional works at: https://scholarsarchive.byu.edu/facpub

Part of the Communication Sciences and Disorders Commons

\section{Original Publication Citation}

Dromey, C., Jang, G-O, \& Hollis, K. (2013) Assessing correlations between lingual movements and formants. Speech Communication, 55, 315-328.

\section{BYU ScholarsArchive Citation}

Dromey, Christopher; Jang, Gwi-Ok; and Hollis, Kristi, "Assessing Correlations Between Lingual Movements and Formants" (2013). Faculty Publications. 1787.

https://scholarsarchive.byu.edu/facpub/1787 
Assessing correlations between lingual movements and formants

Christopher Dromey, PhD

Gwi-Ok Jang, MS

Kristi Hollis, MS

Department of Communication Disorders

Brigham Young University

Corresponding Author

Christopher Dromey, PhD

Department of Communication Disorders

133 John Taylor Building

Brigham Young University

Provo, UT 84602

USA

dromey@byu.edu

tel. $801-422-6461$

fax. 801-422-0197 
Abstract

First and second formant histories have been used in studies of both normal and disordered speech to indirectly measure the activity of the vocal tract. The purpose of the present study was to determine the extent to which formant measures are reflective of lingual movements during diphthong production. Twenty native speakers of American English from the western United States produced four diphthongs in a sentence context while tongue movement was measured with a magnetic tracking system. Correlations were computed between the vertical tongue movements and the first formant, as well as between the anteroposterior movements and the second formant during the transition phase of the diphthong. In many instances the acoustic measures were clearly reflective of the kinematic data. However, there were also exceptions, where the acoustic and kinematic records were not congruent. These instances were evaluated quantitatively and qualitatively in an effort to understand the cause of the discrepancy. Factors such as coarticulation, motor equivalence (including the influence of structures other than the tongue), and nonlinearities in the linkage between movement and acoustics could account for these findings. Recognizing potential influences on the acoustic-kinematic relationship may be valuable in the interpretation of articulatory acoustic data on the individual speaker level.

Keywords: formant, kinematic, diphthong, articulation 


\section{Introduction}

Speech production can be examined in a variety of ways, depending on the goals of the researcher. Perceptual evaluation offers the two key advantages of requiring no instrumentation and being closely linked to the experiences of everyday speakers and listeners. However, because of limitations in the precision and reliability of human perceptual performance, instrumental means of measuring speech have become a mainstay of research in our field [1].

Acoustic analysis allows the noninvasive measurement of many aspects of voice and speech production. When recordings are made with high quality equipment in a quiet environment, it is relatively straightforward to generate sensitive indexes of phonation and articulation. Measures of phonatory function have application not only in research; they are also easily derived from clinical recordings in order to quantify dysphonia severity or treatmentrelated change. As such, they complement the clinician's perceptual evaluation by providing objective evidence of the effects of intervention [2].

The identification of articulatory movements from time-varying acoustic parameters is far more time-consuming than computing perturbation measures from sustained vowel phonation. As a consequence, parameters such as formant transitions, voice onset time, and segment durations are typically not measured in everyday clinical practice. Rather, they are used in basic and clinical research in order to indirectly reveal aspects of the speech movements that underlie sound production. Patterns of change in the first and second formants (F1, F2) have been used in numerous studies to reflect the activity of the vocal tract [e.g, 3,4].

The transfer function of the vocal tract filter depends on the interaction of multiple resonating cavities, which prevents the straightforward identification of the anatomic origin of a particular formant. Nevertheless, the generally accepted view is that F1 is strongly influenced by 
the height of the tongue and jaw and F2 is to a large extent linked to tongue advancement in the mouth. Changes in overall vocal tract length, which result from adjustments to laryngeal height and lip rounding, influence the frequencies of all formants.

The fact that changes in the formant frequencies reflect adjustments to the vocal tract configuration has led to the widespread use of formant histories as indicators of articulatory activity. Weismer and colleagues [5] reported that speakers with amyotrophic lateral sclerosis had shallower formant transition slopes than healthy speakers, and that those with the poorest intelligibility in many instances had trajectories that were nearly flat. Weismer and Berry [6] examined formant trajectories at different speaking rates and found that there was not a linear compression or expansion of the transitions as individuals spoke more quickly or slowly.

F1 and F2 have also been used in the computation of vowel triangles or quadrangles, which reflect the extent of vocal tract movements in the production of corner vowels. Tjaden and Wilding [7] investigated the effects of loud or slow speech on the vowel space area of speakers with dysarthria and found that slower speech led to an expansion of the area, presumably because the articulators had more time to reach their spatial targets. The same study found that formant slopes in diphthongs did not change uniformly with rate or loudness adjustments.

In addition to reflecting certain features of articulatory behavior, formants have also been used as an index of vocal tract steadiness in spasmodic dysphonia or hyperkinetic dysarthria [811]. In this context they have been valuable in characterizing the impact of a disorder on the vocal tract filter as distinct from the laryngeal sound source.

Acoustic analysis of speech is appealing because it is non-invasive and thus has no impact on the way words are spoken. However, there is a degree of ambiguity in the interpretation of the acoustic signal, since a given acoustic change can result from the 
contributions of several structures. This is one of the reasons why some researchers have turned their attention to the movements themselves that underlie the acoustic signal.

Kinematic analysis examines articulatory patterns by tracking the movement of articulators, such as the jaw, lips, and tongue. A number of devices have been developed over the last few decades, and these have enabled researchers to measure the movements of the articulators in both normal and disordered speech. Barlow, Cole, and Abbs [12] suggested a direct examination of disordered speech movements rather than only considering the acoustic result of these movements, because impaired control of muscle contraction and movement is the more direct consequence of neuromotor abnormality. Thus, kinematic analysis brings us a step closer to the disorder itself, removing the intermediate step of acoustic analysis.

Researchers have relied on a variety of techniques to reveal details of articulatory motion. Strain gauges and optical tracking systems $[12,13]$ are relatively straightforward to use, but can only reveal the movements of visible structures. The collection of lingual kinematic data requires more invasive technologies. Using cinefluorography exposes participants to the danger of radiation. The complexity and cost of computerized X-ray microbeam, magnetic resonance imaging (MRI) tagging, and ultrasound systems have limited their use to a few specialized laboratories [14]. Electromagnetic articulography [15-17] has increased in popularity in recent years, although the systems are expensive and require careful setup for experimental use.

A jaw-tracking system that uses a single, permanent magnet has been used to quantify tongue movements during speech $[14,18]$. While this system is limited to tracking a single flesh point on the tongue, its ease of set-up and modest cost allow its application where more sophisticated systems are either unavailable or impractical. 
Because acoustic analysis focuses on the audible results of vocal tract activity, while kinematic approaches reveal details of the underlying movements, it would be valuable to examine the relationship between the data from these two signal sources. As noted earlier, F1 is understood to reflect the vertical movement of the tongue, and F2 its anteroposterior movement [19]. There are several factors that could potentially influence the relationship between these two types of signal. One of these would be motor equivalence [20], whereby slightly different vocal tract configurations across individual speakers can result in the production of a perceptually equivalent phoneme. In other words, there can be more than one way to produce a similar result as movement in one part of the vocal tract co-occurs with adjustments elsewhere that combine to produce the target sound. A further influence on the acoustic-kinematic linkage would be coarticulation, because lingual movements for target sounds can be influenced by the production of neighboring sounds in the context of words or sentences [21-23]. Finally, according to the quantal theory of speech $[24,25]$, the relationship between acoustic and kinematic parameters is not always linear, in that a given degree of articulator displacement in one position may have a much larger influence on acoustics than a similar displacement at a different point along its trajectory. The goal of the present study was to compare the patterns in the first two formants with the magnetically tracked movements of the tongue that they are assumed to represent. Pearson correlation coefficients between the formant and kinematic records were computed during the diphthong transition to evaluate the extent to which the acoustic measures were reflective of the movements. Of particular interest were cases where the acoustic and kinematic data were not predictably correlated. 
2. Method

\subsection{Participants}

Twenty individuals with normal speech (as judged by the experimenters) took part in this study. Nine of the speakers had multiple sclerosis, and had initially been recruited as part of larger study to investigate time-of-day effects on speech production [26,27]. There were 7 women and 2 men, and their ages ranged from 29 to 54 years (mean 42.1). However, when they came to the lab for the study it became immediately apparent that their conversational speech was perceptually normal. Two men with MS and dysarthric speech from the original group [27] are not included in the present report. The remaining 11 participants ( 7 women and 4 men, ages 26 to 61 years, mean 43.9) had a history of normal speech and no neurological disease. All were native speakers of American English from the western United States without any identifiable regional dialect.

\subsection{Speech Tasks}

Participants completed four speech tasks in the following order: maximum sustained vowel phonation, diadochokinetic syllable repetition, sentence production, and a reading passage. For the purposes of the present report only sentence repetition will be considered. Each participant was asked to read two sentences at a normal speech rate and loudness: The boot on top is packed to keep and The boy gave a shout at the sight of the cake. Although only three repetitions were used in the present analysis, five repetitions were recorded to allow for the replacement of any disfluent or misarticulated tokens. The sentences were selected because they

included corner vowels in the first and a series of diphthongs in the second. The second sentence 
was the focus of the present study because it allowed a comparison of lingual movement with formant transitions during the diphthongs.

\subsection{Equipment}

During each speech task, the acoustic signal was recorded into a Dell computer via a microphone (AKG C $2000 \mathrm{~B}$ ) that was positioned $15 \mathrm{~cm}$ from the speaker's mouth. The acoustic signal passed through a Samson Mix Pad 4 preamplifier and then a Frequency Devices 9002 low pass filter (20 kHz cutoff). An adapted BioResearch Associates JT-3 jaw tracking instrument was used for measuring tongue movement as described in a study by Dromey and colleagues [14]. This device has analog outputs for vertical and anteroposterior movements, referenced to the headset. A Windaq 720 multi-channel analog-to-digital conversion system was used to digitize the acoustic signal from the microphone and the output of the magnetic tracking system. The microphone signal was sampled at $44.1 \mathrm{kHz}$ and the kinematic signals at $1 \mathrm{kHz}$.

\subsection{Procedure}

Each participant sat in an Acoustic Systems sound booth on a chair in front of the microphone. Using cyanoacrylate glue, the researcher attached a small magnet to the upper surface of the participant's tongue at midline, approximately $1 \mathrm{~cm}$ posterior to the tip. Sentence and passage reading materials were provided on a stand in front of the participants.

\subsection{Data Analysis}

The multi-channel file which included the audio and magnet movement data was opened in the Windaq Waveform Browser application (version 2.49; DATAQ Instruments, 2006). Within the longer recording of the session, this file contained five repetitions of the sentence The 
boy gave a shout at the sight of the cake. The first three repetitions were selected for analysis. From the recorded channels, only those for the audio, vertical and anteroposterior magnet movements were exported as a new binary file. This new file was then imported into a custom application in MATLAB [28], which allowed the researcher to segment the individual diphthongs, /or/, /au/, /ar/, and /er/ from the time-aligned audio and magnet movement data. Each diphthong was visually segmented from the audio recording (confirmed by listening and examination on a spectrogram). The audio channel of each segmented diphthong was saved into a short wav file (at the original sample rate of $44.1 \mathrm{kHz}$ ), and these files were subsequently imported into PRAAT software [version $5.0 .47 ; 29$ ] to compute F1 and F2 via LPC analysis. The Burg method was used with a $6 \mathrm{~dB}$ per octave pre-emphasis from $50 \mathrm{~Hz}$. The display was adjusted to show 3 formants with a window length of $25 \mathrm{~ms}$ and a dynamic range of $30 \mathrm{~dB}$. Because individual speakers varied in their acoustic characteristics, the maximum formant frequency was adjusted between 2600 and $4800 \mathrm{~Hz}$ to maximize the visually-judged accuracy of formant tracking on the spectrogram. The most accurate tracking was achieved with lower maximum frequencies for /os/ and /av/, and higher values for /ai/, and /es/. PRAAT created a text file listing the formant values from the start to the end of each diphthong at $1 \mathrm{~ms}$ intervals, thus corresponding to and aligned with the kinematic record.

These text files were then imported back into MATLAB, and the F1 and F2 formant values were time-aligned with the vertical and anteroposterior magnet movement records. The kinematic data were not rotated; the coordinate plane was relative to the headset. While the vertical dimension was approximately perpendicular to the occlusal plane, this correspondence was not formally determined. Both formant and kinematic records were low-pass filtered at 10 $\mathrm{Hz}$ prior to their comparison to remove higher frequency noise. Finally, Pearson correlations 
between F1 and the magnet's vertical movement and between F2 and anteroposterior movement were computed for the individual diphthongs. A continuous correlation function [30] was computed by means of a sliding 30 sample window that computed the correlation between the acoustic and kinematic movement records point-by-point along the entire diphthong. The correlation was computed from a point before the diphthong began to another after its end to avoid the need for end-padding the 30 sample window within the transition. The continuous correlation trace allowed an observation of changes in the relationship between the two signals over time. Figures 1 and 2 show the formant histories and tongue marker position signals for two different diphthongs and also the temporal segmentation approach that was used in the present study. The diphthong was divided into four equal periods of time. The first and last quartiles were operationally defined as the diphthong onset and offset respectively. The middle two were defined as the diphthong transition. This operational approach was used to avoid the potential for human measurement bias during signal analysis. The focus of the analysis was on the diphthong transition, given that a greater movement would be expected during this phase. Figure 1 shows signals from the word boy, where the formant transitions during the diphthong were readily captured by the operationally defined middle two quartiles of the acoustically and perceptually segmented diphthong. Figure 2, on the other hand, shows the word shout, where the lingual movement records only show the anticipated movements during the offset phase. The potential role of coarticulation of this sound will be considered in greater detail in the discussion (section 4.1.2).

Several dependent measures were computed for each diphthong. These included the mean correlation (F1 vs. vertical movement, F2 vs. anteroposterior movement) during the diphthong transition, the transition duration (ms), the F1 and F2 transition extent (maximum minus 
minimum formant frequency in $\mathrm{Hz}$ ), and the vertical or anteroposterior magnet transition displacement in $\mathrm{mm}$.

\subsection{Statistical Analysis}

Mean values of the dependent variables for the three analyzed diphthongs were calculated for each sound and each speaker. Correlation coefficients were computed between the acoustickinematic correlations themselves and the other dependent measures in order to evaluate the impact of movement size, formant transition extent, and transition duration on the strength of the acoustic-kinematic correlation. Because correlation coefficients are not normally distributed, they were first Fisher-z transformed before being correlated with the other variables. All statistical testing was completed with IBM-SPSS 20 [31].

\section{Results}

Because no significant differences were found between the speakers with MS and the control group for these speakers in an earlier study [26], the results represent the combined data from both groups. As noted above, all participants had perceptually normal speech, as judged by the experimenters. T-tests comparing the acoustic-kinematic correlations for men and women showed no differences, thus all further testing was done for the 20 speakers combined.

Figure 3 shows the mean (of three tokens) individual speaker acoustic-kinematic correlations for all four diphthong transitions, with vertical movements in the left column and anteroposterior movements in the right column of plots. 


\subsection{Relationship between F1 and Vertical Tongue Movement}

Table 1 shows the means and standard deviations, along with the minimum and maximum values of the dependent variables for vertical movements across the 20 speakers for each diphthong. A negative correlation would be predicted between the formant and magnet signals because F1 should decrease as the tongue position elevates during the production of each diphthong.

For the /oI/ transition only one of the 20 speakers displayed the predicted strong, negative correlation between the acoustic and kinematic signals. The mean correlation was .070 , and the individual speakers' correlations covered a range of values, both positive and negative. This diphthong transition had the longest mean duration $(92.1 \mathrm{~ms})$ of the four sounds in the study, and it also had the largest mean vertical displacement $(2.87 \mathrm{~mm})$.

For /av/, 15 of the 20 speakers exhibited a negative correlation, although only five had a Pearson $r$ value that was more negative than -.500 . The mean correlation of -.268 indicated a modest negative association between the acoustic and kinematic variables when all speakers were considered as a group.

During the production of /ai/ all 20 speakers had a negative correlation during the transition. The strength of the negative correlation varied across speakers, although the mean value of -.727 indicated that the predicted relationship between the kinematic and acoustic variables was present for this sound. Fifteen of the speakers had a Pearson $r$ value that was more negative than -.500 . The mean vertical magnet displacement was similar for /av/ and /ai/ (just over $1.5 \mathrm{~mm}$ for each). 
For /eI/, 16 of the 20 speakers had a negative correlation, with a group mean of -.345 . For 10 of the individuals the Pearson $r$ value was more negative than -.500 . The mean transition duration for this sound was the briefest of the four diphthongs $(55.1 \mathrm{~ms})$ and the vertical displacement of the magnet was the smallest $(0.41 \mathrm{~mm})$. Likewise, the mean extent of $\mathrm{F} 1$ change during the transition was the smallest $(78.6 \mathrm{~Hz})$.

\subsection{Relationship between F2 and Anteroposterior Tongue Movement}

Table 2 shows the means and standard deviations, along with the minimum and maximum values of the dependent variables for anteroposterior movements across the 20 speakers for each diphthong. A positive correlation was predicted for the relationship between F2 and anteroposterior tongue movement because F2 should increase as the tongue moves forward.

During the production of /oI/, 19 of the 20 participants displayed a positive correlation, with values exceeding .820 . The mean value of .899 represented a strong association between the acoustic and kinematic variables for this diphthong transition. The mean anteroposterior displacement (3.89 $\mathrm{mm}$ ) was greatest for this diphthong, as was the mean change in F2 frequency $(931.5 \mathrm{~Hz})$ during the transition.

For /av/, 17 of the 20 speakers exhibited a positive correlation, which contributed to a mean Pearson r value of .470. For 10 individuals, the $r$ was greater than .500 . The mean anteroposterior displacement for /av/ $(1.87 \mathrm{~mm})$ was greater than for /ai/ $(1.61 \mathrm{~mm})$, which was unexpected, given that the movement from a lower to a higher back vowel position had been anticipated to be primarily vertical.

During /aI/ production the mean correlation of .318 reflected a majority of positive correlations (16 of 20 speakers), but also a wide range in the strength of the association between 
the acoustic and kinematic measures. Although the anteroposterior displacement was slightly smaller for this sound than for /av/, the mean change in F2 was greater $(442.3 \mathrm{~Hz})$.

During the production of /es/ there was a range of positive correlations for 18 of 20 speakers, which contributed to a group mean of .569 .14 of the speakers had a correlation stronger than .500 . The mean anteroposterior displacement was the smallest for this sound (0.66 $\mathrm{mm})$, and the extent of $\mathrm{F} 2$ change was also modest $(111 \mathrm{~Hz})$.

\subsection{Factors Linked to Acoustic-Kinematic Correlations}

It is notable from Tables 1 and 2 that while all speakers produced perceptually accurate diphthongs, there was a wide range between the minimum and maximum values on many of the dependent measures. This observation led to an examination of the extent to which the size of the recorded movements, the duration of the transition, and the extent of the changes in F1 and F2 might be related to the strength of the correlation between them.

First, the acoustic-kinematic correlations were Fisher-z transformed (because correlations are not normally distributed) so that they could themselves be correlated with the displacement, duration, and formant change variables. Then the individual diphthongs were analyzed to determine the degree to which correlation strength was associated with the other measures.

\subsubsection{F1/vertical correlations and their association with other variables}

For / II/, the F1/vertical correlation was negatively correlated with vertical displacement (r $=-.463, \mathrm{p}=.04)$, although once the outlier visible in the top left panel of Figure 3 was removed, the correlation was no longer significant. 
For /av/, the F1/vertical correlation was correlated with the duration of the transition ( $\mathrm{r}=$ $.525, \mathrm{p}=.017)$. The predicted negative correlation between tongue elevation and F1 decrease was stronger for the briefer diphthong transitions, but there was no association with the distance traveled or the $\mathrm{F} 1$ change in $\mathrm{Hz}$.

For the diphthong /ar/, the F1/vertical correlation was not associated with any pattern of change in the other variables. Even though all of the correlations followed the negative trend, the strength of this correlation was not predictable based on the movement itself or the resultant acoustic signal.

For /es/, the F1/vertical correlation was correlated with the vertical displacement of the magnet $(\mathrm{r}=-.563, \mathrm{p}=.010)$. More strongly negative correlations were associated with larger vertical movements.

3.3.2 F2/anteroposterior correlations and their association with other variables

For /ol/, the F2/anteroposterior correlation was associated with the horizontal displacement of the magnet $(\mathrm{r}=.511, \mathrm{p}=.021)$. Stronger correlations were linked to larger movements of the magnet.

For /av/, the strength of the F2/anteroposterior correlation was negatively correlated with the duration of the diphthong transition $(\mathrm{r}=-.451, \mathrm{p}=.046)$. The correlation was also associated with the horizontal displacement of the magnet $(\mathrm{r}=.516, \mathrm{p}=.020)$. The stronger F2/anteroposterior correlations were found for larger magnet movements and briefer transition durations. 
For /aI/ there was no association between the F2/anteroposterior correlation and the other variables.

For /ei/, the F2/anteroposterior correlation was positively associated with the horizontal displacement of the magnet $(\mathrm{r}=.703, \mathrm{p}=.001)$. Larger movements were linked to stronger correlations.

\subsection{Qualitative Examination of Kinematic and Formant Records}

While Figure 3 shows the mean correlations for three tokens of each diphthong produced by the speakers, it does not show qualitatively how the correlation often fluctuated during the production of the sound. Figures 1 and 2 reveal patterns seen throughout the dataset, namely that the continuous correlation trace tended to switch between a highly positive or highly negative value, rather than hovering at some intermediate level. For example, during the transition segment in Figure 2, there is a period around $170 \mathrm{~ms}$ where the otherwise negative F1/vertical correlation briefly becomes positive. Figure 2 also shows how the F2/anteroposterior correlation during the transition began as a negative value and then at around $125 \mathrm{~ms}$ became positive for the rest of the transition.

In addition to considering the mean acoustic-kinematic correlations across speakers and sounds, individual speakers' data were examined where there was substantial inconsistency in the correlation from token to token as revealed by a high coefficient of variation (standard deviation of the three tokens, divided by the mean). In general it was found that the kinematic trajectories were relatively consistent while the formant histories were more prone to variability, although this was not always the case. Several examples will serve to illustrate this finding.

Figure 4 shows three examples of tokens from individual speakers where the three diphthong repetitions resulted in variability in the acoustic-kinematic correlation. The $x-y$ plots 
show the kinematic trajectory on the left and the corresponding movement in formant space on the right for the diphthong transition (thus, not including the diphthong onsets or offsets). All plots in this figure are for the diphthong /av/. In the upper panels are plots for Speaker 7, for whom the lingual movements were fairly simple and consistent, whereas the formant tracks were curved and irregular. As noted above, it was more often the case that the tongue movements followed a similar pattern across repetitions, while the formants were more likely to vary. A less common pattern was for greater variability in the kinematic than the acoustic signal. The middle panels of Figure 4 show three repetitions for Speaker 4. Here, the lingual movements, while sharing a similar shape, were variable in their absolute locations; the formant tracks were relatively consistent by comparison. The least common occurrence (only found twice in the entire dataset) was when individual tokens differed markedly in their acoustic-kinematic correlation because the formant tracks were fairly similar across tokens, but the kinematic histories were more variable in their form as well as location. The lower plots show how this occurred for Speaker 4.

\section{Discussion}

The purpose of this study was to investigate the correlation between acoustic and kinematic variables in perceptually normal speech during the production of four diphthongs in a sentence context. In many instances the association between the two data sets followed the pattern that would be predicted, based on typically accepted notions of the movements that contribute to changes in F1 and F2. However, there were also a number of exceptions that warrant further consideration, especially for F1. In the sections below, the primary focus will be 
on those instances where the formant histories did not appear to follow the movement of the tongue in a predictable way.

\subsection{Acoustic-Kinematic Relationships}

4.1.1 Correlations for /oI/. The frequency of F1 during the diphthong transition did not follow the vertical tongue movement in the predicted fashion because the anticipated negative correlation between the acoustic and kinematic variables was not found. Instead, with only one exception among the 20 speakers, the correlation was either positive or close to zero. The widely held view is that F1 decreases as the tongue is elevated because this movement enlarges the pharyngeal space where F1 resonates. However, this general principle may be too simplistic to account for other acoustic interactions that influence the frequency of F1 during the production of /oI / in the present context. It has been well established that coarticulatory influences can cause the production of a target sound to change [21-23]. Thus, the diphthongs in the sentence context of the present study may have been produced differently than the same sounds spoken in isolation. One potential explanation for the absence of the predicted correlation would be that the closed vocal tract for /b/ may have led to a low F1 as the diphthong began, which then increased slightly in frequency as the vocal tract opened, then decreased again as the tongue rose towards the end of the diphthong. The curved F1 history for /oI/in Figure 1 shows how F1 increased and then decreased during the transition, while the magnet movement followed a simpler anterior and vertical course. These plots further suggest that the movement of the anterior tongue was not the only contributor to changes in F1. The diphthong /or/ would typically begin with the lips being somewhat rounded, subsequently retracting during the transition to the second vowel in the 
diphthong. Since lip shape and position data were not collected in the present study, it would be valuable in future research to examine these changes in connection with the acoustic output to learn whether they correlate more systematically with F1 in this context.

In contrast to the relationship between F1 and vertical movement, F2 clearly followed the anteroposterior tongue movement because it showed an overall strong positive correlation with the magnet's movement, and this correlation was stronger for larger movements. As a general statistical principle, correlations tend to be stronger for data with a wider range [32], and the size of the horizontal displacement for this sound may have contributed to this effect.

4.1.2 Correlations for /av/. Averaged across the speakers, F1 and the vertical tongue movement showed a modest negative correlation during the transition. Individual speakers varied substantially in the strength of the acoustic-kinematic correlation. Although the distance moved by the magnet did not account for the strength of the correlation, the transition duration did. It is not immediately clear why a briefer transition would be associated with a stronger linkage between the acoustic and kinematic signals, unless there was less time for changes in lip shape to potentially influence the acoustics. As noted above, further studies that include the measurement of lip movements could shed valuable light on this question, as well as reveal important differences between speakers. Motor equivalence across individuals has been documented in previous research [20], whereby a perceptually acceptable sound can be formed in more than one way. It is possible that in the present study, speakers who produced this diphthong transition quickly did so without as much contribution from the lips.

When /av/ is produced in the context of the word shout, the anterior tongue placement for $/ S /$ is followed by a backward movement for $/ a /$ and $/ \mho /$. This can be seen during the diphthong transition in Figure 2, where a more negative displacement value reflects a more posterior 
position. Thus, coarticulatory influences might have led to a greater anteroposterior tongue movement for this sound than when it is produced in isolation. Far from being a primarily vertical transition (as might be anticipated from the phonetic transcription), the anteroposterior movement on average exceeded the upward displacement, and the curved kinematic trajectory seen in the middle and lower panels of Figure 4 was found extensively during visual inspection of individual tokens across the speakers. In Figure 2, the transitional lingual movement between the two vowels in the diphthong occurred later than during the operationally defined central two quartiles. It would be valuable to compare the same diphthong produced in isolation to parse out the influence of coarticulation.

4.1.3 Correlations for /aI/. For this sound, F1 tracked the vertical tongue movement quite well because strong negative correlations were found for most of the speakers. The relatively large vertical tongue movement (second only to /or/) might have been a factor in this finding, given the potential for data range to influence correlation. However, the size of the displacement did not correlate with the strength of the acoustic-kinematic correlation across speakers. It could be tentatively speculated that the tongue was moving through a range where linearity governed the link between movement and the resulting sound characteristics, whereas the other diphthongs may have involved movements where the linkage was far less linear for F1. Another explanation may lie in the lack of lip protrusion that would be anticipated for both of the sounds in this diphthong. Without the influence of rounding and protrusion, the movement of the tongue may have been the strongest contributor to F1 change in this context.

Contrary to the theoretical prediction, F2 did not unambiguously reflect the anteroposterior tongue movement. Most individuals showed a positive correlation during the transition, but the strength of the correlation was highly variable. The measures of displacement, 
transition extent in $\mathrm{Hz}$, and duration were not systematically linked to the strength of the correlation. Thus, the reason for these differences across speakers is unclear. Visual inspection of the kinematic records revealed curved trajectories for this sound, which may be linked to the alveolar articulatory position for /s/ immediately prior to the low back starting vowel for this diphthong. It is also possible that differences in speaking style that are not critical to listeners' comprehension could have contributed to the variability across individuals in the acoustickinematic correlation.

4.1.4 Correlations for /eI/. F1 tracked the vertical tongue movement for most of the speakers in the study. Since the vertical displacement was very small for this sound, a strong correlation would not have been predicted. However, the association between vertical displacement and the strength of the acoustic-kinematic correlation revealed that even though the largest of the speakers' movements was small compared to other sounds, larger movements were more strongly correlated with F1 changes.

As with F1, the F2-kinematic correlation was stronger for larger lingual movements. The phonetic symbol for this sound would suggest a primarily vertical trajectory, but the magnet data reveal on average a larger anteroposterior displacement during the transition. Visual inspection revealed a simple and relatively straight tongue movement pattern for most speakers (with only a few whose durations were longer showing a slightly curved trajectory). The more common trajectory is understandable, given that this sound had the briefest transition duration of the four diphthongs, and there can be tendency for some speakers to produce this phoneme more like a monophthongal vowel than a diphthong.

\subsection{Qualitative Observations}


Figures 1 and 2 reveal a number of important features of the relationship between the acoustic and kinematic records. As noted earlier, the correlations tended to fluctuate between the extremes of positive and negative values. For example, the vertical acoustic-kinematic correlation shown for /av/ in Figure 2 began the transition as strongly negative, then briefly became positive before returning to a negative value. Examination of the two middle panels reveals that the lingual magnet was mostly descending during this transition (a somewhat surprising observation in itself, but likely due to coarticulation following $/ \int /$ ), but the F1 track rose and fell. As a result there was a time during which the direction of change was the same for both signals, resulting in the positive correlation. Figure 2 also shows how F2 tracked the anterioposterior magnet movement as predicted from about a third of the way through the transition, and even throughout the operationally defined diphthong offset. In this instance, the working definition of the diphthong transition did not capture the anticipated lingual elevation from the first to the second vowel in /av/. The vertical magnet movement did not begin until the operationally defined transition was almost over, leaving most of the ascent for the presumed 'steady state' at the end of this diphthong. Thus, the assumption that middle $50 \%$ of the diphthong would represent the transition is not well supported by the data in this particular example.

\subsection{General Discussion}

Some of the findings of the present study might be considered in the context of the quantal theory of speech $[24,25]$. According to this theory, there are regions of articulatory movement for which there is little change in the acoustic measures. Conversely, there can be 
other regions along the movement trajectory where even a small change in position makes a large acoustic difference. As Stevens explains, "discontinuous attributes of the acoustic signal occur in spite of rather continuous movements or changes in the articulatory parameters" (p. 5). Stevens proposed that there are various factors, such as the place of constriction or lip rounding, which can lead to a quantal relationship between acoustic and kinematic parameters. In the current study, it could be speculated that even though tongue movements were continuous, at some point the formant frequency was more sensitive to tongue movement and thus changed more substantially. Pearson correlations reflect the strength of a linear association, and it is possible that other types of nonlinear analysis might prove valuable in future studies. Also, since lip rounding was not measured in the present study, its influence may have been significant in those instances where the formant changes differed from the hypothesized patterns.

Another factor that may account for some of the unpredicted correlations between acoustics and kinematics in the present study is the anatomic variability in individuals' vocal tract structures. As Kent et al. [1] noted, since formants depend in part on the length of the vocal tract, an individual speaker's anatomic characteristics should be considered. The size and shape of the articulators, such as tongue, hard palate, pharynx, lips, jaw, and teeth can vary across individuals, and thus a given lingual movement may not have the same acoustic result across speakers. If an individual has a proportionally larger tongue, its range of motion may not be equivalent to that of another individual with smaller structures. Since the movement of the tongue at midline was the only kinematic data source in the study, it cannot be determined how much any movements of the lingual edge might have contributed to the acoustics. Ultrasound and EPG studies have revealed that the tongue surface can be shaped in a variety of ways during 
vowel production [33], and the extent to which individual differences in the surface shape may have contributed to the acoustics in the present study is not known.

The movement of vocal tract structures other than in the oral cavity may also have influenced the formant frequencies. For example, raising the larynx or constricting the pharynx would be expected to raise formant frequencies. These movements would not be reflected in the position of the magnet attached to the anterior tongue, and thus may have influenced the kinematic/acoustic correlations in the present study. The limitations of tracking a single flesh point are not trivial, and future studies that measure the movements of multiple points on the tongue, as well as other articulators, will allow a clearer understanding of the links between movement and acoustics.

Individuals' idiosyncratic articulatory patterns could also have influenced the acoustickinematic associations in the current data set. The ability of the vocal tract to achieve an equivalent acoustic output from slightly different articulatory movements - motor equivalence could have contributed to the present findings [20]. Even if individuals do not move a given articulator the same way to produce a certain sound, other vocal tract adjustments can compensate to produce the target sound accurately. Thus, in this study it is possible that the nature of the tongue movements could have varied across speakers even where the acoustic output was similar.

One of the limitations of the current study lay in the process of operationally defining the onset, transition, and offset of each diphthong. In order to avoid the influence of subjective segmentation judgments during data analysis, the audio recording was automatically divided into 4 equally-spaced segments. This operational definition of the three parts of the diphthong may have been overly simplistic. However, the automatic segmentation was reasoned to be a 
necessary process for this study because reliably identifiable segments were required in order to quantify and compare the two physically different signals from the kinematic and acoustic sources. Tasko and Westbury [34] described some important differences between acoustic and kinematic segmentation, including the observation that lingual movement is nearly continuous, even across acoustically-defined segments that can be identified on the basis of phoneme boundaries on a spectrogram. It may be valuable in future work to consider acoustic-kinematic linkages within speech segments that are selected on the basis of criteria other than the operational definition used in the present research.

A further limitation of the present study is the potential for formant tracking errors during software analysis of the audio signal. Some speech recordings are easy to analyze, because the formants stand out clearly on a spectrogram and the software is able to identify the spectral prominence for each formant. Within a group of normal speakers, some will have breathier voices or broader formant bandwidths, which can occasionally make their vowels and diphthongs harder to analyze. Low-pass filtering was used on the formant histories to smooth out minor fluctuations before they were correlated with the kinematic record, but this would not have eliminated the potential for errors in formant identification in Praat.

Although there were numerous instances where the formants did not track the lingual movements in a consistent, predictable way, a number of patterns did emerge that reinforce the assumption that $\mathrm{F} 1$ is sensitive to vertical and $\mathrm{F} 2$ to anteroposterior tongue movement. A perusal of Figure 3 reveals that there was a relatively strong association between F1 and vertical movement for several speakers for /av/, for many speakers for /eI/, and for nearly all speakers for /aI/. This latter diphthong was flanked by alveolar consonants, and its component vowels did not involve any lip rounding. These factors may have contributed to a relatively straightforward 
association between tongue height and F1 that was possibly masked by bilabial consonant closure or vowel lip-rounding in some of the other sounds examined in this study. For /ai/ the strength of the acoustic-kinematic association was robust across movements that differed in duration or displacement, whereas for /es/ the correlation increased for larger movements. Briefer durations led to a stronger F1/movement correlation for /av/.

Figure 3 also shows that F2 was highly reflective of anteroposterior tongue movement for /or/, with larger displacements showing a stronger correlation. The same principle applies to /eI/, even though the movements on average were quite small. For the sounds /av/ and /ar/, even though several speakers showed weak or unpredicted acoustic-kinematic correlations, there were more cases that followed the predicted pattern, indicating that F2 in many cases tracked lingual advancement reasonably well during diphthong production.

\subsection{Conclusion}

It is generally accepted that changes in the formant frequencies of vowels and diphthongs indirectly reflect tongue movements; F1 reflects vertical displacement and F2 primarily reflects anteroposterior movement. Accordingly, the present study was performed to test the strength and consistency of this association. The results of the current study showed that in a number of instances, the formants reliably tracked the tongue movements, but in others the association was far less clear. In other words, there was variability across individuals and sounds in the strength of the correlations between the acoustic and kinematic variables. These findings suggest that assumptions about the relationship between the formants and tongue movements may be overly simplistic, and in connected speech their relationship can be more complex. Therefore, 
researchers should exercise caution when interpreting acoustic measures of lingual activity for an individual speaker, given the potential contributions of coarticulation, motor equivalence, and the contributions of other vocal tract structures to the production of these sounds.

\section{Acknowledgements}

We express appreciation to the individuals who volunteered their time to participate in this study. Funding was provided by the David O. McKay School of Education at Brigham Young University. This paper was based on the master's theses of the second and third authors. 


\section{Reference List}

[1] R. D. Kent, G. Weismer, J. F. Kent, H. K. Vorperian, and J. R. Duffy, Acoustic studies of dysarthric speech: Methods, progress and potential, J Commun Disord, 32 (1999) 141-186.

[2] A. S. Dagli, H. F. Mahieu, and J. M. Festen, Quantitative analysis of voice quality in early glottic laryngeal carcinomas treated with radiotherapy, Eur Arch Otorhinolaryngol, 254 (1997) 78-80.

[3] C. Dromey, S. Nissen, N. Roy, and R. M. Merrill, Articulatory changes following treatment of muscle tension dysphonia: Preliminary acoustic evidence, Journal of Speech, Language, and Hearing Research, 51 (2008) 196-208.

[4] J. M. Liss and G. Weismer, Qualitative acoustic analysis in the study of motor speech disorders, J Acoust Soc Am, 92 (1992) 2984-2987.

[5] G. Weismer, R. Martin, R. D. Kent, and J. F. Kent, Formant trajectory characteristics of males with amyotrophic lateral sclerosis, J. Acoust. Soc. Am., 91 (1992) 1085-1098.

[6] G. Weismer and J. Berry, Effects of speaking rate on second formant trajectories of selected vocalic nuclei, J. Acoust. Soc. Am., 113 (2003) 3362-3378.

[7] K. Tjaden and G. E. Wilding, Rate and loudness manipulations in dysarthria: acoustic and perceptual findings, J. Speech Lang Hear. Res., 47 (2004) 766-783.

[8] M. P. Cannito, Vocal tract steadiness in spasmodic dysphonia, in: K. M. Yorkston and D. R. Beukelman (Eds.), Recent advances in clinical dysarthria, Little, Brown and Co., Boston, 1989, pp. 243-262.

[9] B. Gerratt, Formant frequency fluctuation as an index of motor steadiness in the vocal tract, J. Speech Hear. Res., 26 (1983) 297-304. 
[10] P. Zwirner and G. J. Barnes, Vocal tract steadiness: a measure of phonatory and upper airway motor control during phonation in dysarthria, J. Speech Hear. Res., 35 (1992) 761768.

[11] P. Zwirner, T. Murry, and G. E. Woodson, Effects of botulinum toxin on vocal tract steadiness in patients with spasmodic dysphonia, Eur Arch Otorhinolaryngol, 254 (1997) 391-395.

[12] S. M. Barlow, K. J. Cole, and J. H. Abbs, A new head-mounted lip-jaw movement transduction system for the study of motor speech disorders, J. Speech Hear. Res., 26 (1983) 283-288.

[13] A. Smith, L. Goffman, H. N. Zelaznik, G. Ying, and C. McGillem, Spatiotemporal stability and patterning of speech movement sequences, Exp Brain Res, 104 (1995) 493-501.

[14] C. Dromey, S. Nissen, P. Nohr, and S. G. Fletcher, Measuring tongue movements during speech: Adaptation of a magnetic jaw-tracking system, Speech Communication, 48 (2006) 463-473.

[15] A. S. Mefferd and J. R. Green, Articulatory-to-acoustic relations in response to speaking rate and loudness manipulations, J. Speech Lang Hear. Res., 53 (2010) 1206-1219.

[16] J. S. Perkell, M. H. Cohen, M. A. Svirsky, M. L. Matthies, I. Garabieta, and M. T. T. Jackson, Electromagnetic Midsagittal Articulometer Systems for Transducing Speech Articulatory Movements, J Acoust Soc Am, 92 (1992) 3078-3096.

[17] P. W. Schonle, K. Grabe, P. Wenig, J. Hohne, J. Schrader, and B. Conrad, Electromagnetic articulography: Use of alternating magnetic fields for tracking movements of multiple points inside and outside the vocal tract, Brain Lang, 31 (1987) 26-35. 
[18] S. L. Nissen, C. Dromey, and C. Wheeler, First and second language tongue movements in Spanish and Korean bilingual speakers, Phonetica, 64 (2007) 201-216.

[19] C. T. Ferrand, Speech science: An integrated approach to theory and clinical practice, Allyn and Bacon, Boston 2007.

[20] O. M. Hughes and J. H. Abbs, Labial-mandibular coordination in the production of speech: Implications for the operation of motor equivalence, Phonetica, 33 (1976) 199-221.

[21] P. S. Beddor, A coarticulatory path to sound change, Language, 85 (2009) 785-821.

[22] J. Ohala, Coarticulation and phonology, Language and Speech, 36 (1993) 155-170.

[23] B. H. Story, Vowel and consonant contributions to vocal tract shape, J. Acoust. Soc. Am., 126 (2009) 825-836.

[24] K. N. Stevens, On the quantal nature of speech, Journal of Phonetics, 17 (1989) 3-45.

[25] K. N. Stevens and S. J. Keyser, Quantal theory, enhancement and overlap, Journal of Phonetics, 38 (2010) 10-19.

[26] Jang, G. O. The relationship between acoustic and kinematic measures of diphthong production. 2010. Brigham Young University.

Ref Type: Thesis/Dissertation

[27] Hollis, K. L. The effects of fatigue on acoustic measures of diphthongs in individual with multiple sclerosis. 2009. Brigham Young University.

Ref Type: Thesis/Dissertation

[28] The Mathworks. Matlab. 2009. Natick, MA.

Ref Type: Computer Program

[29] Boersma, P. and Weenink, D. Praat. 2007.

Ref Type: Computer Program 
[30] S. Tingley and C. Dromey, Phonatory-articulatory relationships: Do speakers with spasmodic dysphonia show aberrant lip kinematic profiles? Journal of Medical SpeechLanguage Pathology, 8 (2000) 249-252.

[31] SPSS, Inc. SPSS. 2008.

Ref Type: Computer Program

[32] G. V. Glass and K. D. Hopkins, Statistical methods in education and psychology, Prentice Hall, Englewood Cliffs, NJ 1984.

[33] M. Stone and A. Lundberg, Three-dimensional tongue surface shapes of English consonants and vowels, J Acoust Soc Am, 99 (1996) 3728-3737.

[34] S. M. Tasko and J. R. Westbury, Defining and measuring speech movement events, JSLHR, 45 (2002) 127-142. 
Table 1. Descriptive statistics from all speakers for the dependent variables reflecting vertical lingual movement for the four diphthongs.

\begin{tabular}{|c|c|c|c|c|c|}
\hline Diphthong & Variable & Minimum & Maximum & Mean & St Dev \\
\hline \multirow[t]{4}{*}{ /ог/ } & F1 / Vertical Correlation & -.951 & .546 & .070 & .338 \\
\hline & Transition Duration (ms) & 47.0 & 178.3 & 92.1 & 32.1 \\
\hline & F1 Extent $(\mathrm{Hz})$ & 32.7 & 216.6 & 100.3 & 54.2 \\
\hline & Vertical Displacement (mm) & 1.34 & 4.35 & 2.87 & 0.89 \\
\hline \multirow[t]{4}{*}{ /av/ } & F1 / Vertical Correlation & -.942 & .474 & -.268 & .362 \\
\hline & Transition Duration (ms) & 44.0 & 112.3 & 78.9 & 17.6 \\
\hline & F1 Extent $(\mathrm{Hz})$ & 31.0 & 234.3 & 115.2 & 53.5 \\
\hline & Vertical Displacement (mm) & .15 & 2.40 & 1.56 & .58 \\
\hline \multirow[t]{4}{*}{ /aI/ } & F1 / Vertical Correlation & -.989 & -.037 & -.727 & .287 \\
\hline & Transition Duration (ms) & 36.7 & 88.7 & 61.2 & 14.6 \\
\hline & F1 Extent $(\mathrm{Hz})$ & 13.8 & 347.1 & 164.1 & 78.7 \\
\hline & Vertical Displacement (mm) & .44 & 2.93 & 1.66 & .80 \\
\hline \multirow[t]{4}{*}{ /eI/ } & F1 / Vertical Correlation & -.891 & .423 & -.345 & .405 \\
\hline & Transition Duration (ms) & 39.7 & 82.3 & 55.1 & 13.2 \\
\hline & F1 Extent $(\mathrm{Hz})$ & 11.4 & 201.1 & 78.6 & 49.5 \\
\hline & Vertical Displacement (mm) & .09 & .88 & .41 & .22 \\
\hline
\end{tabular}

Note: St Dev = standard deviation 
Table 2. Descriptive statistics from all speakers for the dependent variables reflecting anteroposterior lingual movement for the four diphthongs.

\begin{tabular}{|c|c|c|c|c|c|}
\hline Diphthong & Variable & Minimum & Maximum & Mean & St Dev \\
\hline \multirow{4}{*}{ /OI/ } & F2 / A-P Correlation & -.212 & .998 & .899 & .266 \\
\hline & Transition Duration (ms) & 47.0 & 178.3 & 92.1 & 32.1 \\
\hline & F2 Extent (Hz) & 535.0 & 1613.7 & 931.5 & 264.7 \\
\hline & A-P Displacement (mm) & 1.24 & 6.25 & 3.89 & 1.37 \\
\hline \multirow[t]{4}{*}{ /av/ } & F2 / A-P Correlation & -.605 & .998 & .470 & .461 \\
\hline & Transition Duration (ms) & 44.0 & 112.3 & 78.9 & 17.6 \\
\hline & F2 Extent $(\mathrm{Hz})$ & 99.1 & 505.0 & 254.2 & 106.3 \\
\hline & A-P Displacement (mm) & .34 & 3.13 & 1.87 & .81 \\
\hline \multirow[t]{4}{*}{ /aI/ } & F2 / A-P Correlation & -.952 & .969 & .318 & .508 \\
\hline & Transition Duration (ms) & 36.7 & 88.7 & 61.2 & 14.6 \\
\hline & F2 Extent $(\mathrm{Hz})$ & 128.8 & 728.3 & 442.3 & 159.2 \\
\hline & A-P Displacement (mm) & .23 & 3.88 & 1.61 & .94 \\
\hline \multirow[t]{4}{*}{ /eI/ } & F2 / A-P Correlation & -.333 & .997 & .569 & .382 \\
\hline & Transition Duration (ms) & 39.7 & 82.3 & 55.1 & 13.2 \\
\hline & F2 Extent $(\mathrm{Hz})$ & 23.1 & 261.8 & 111.0 & 69.0 \\
\hline & A-P Displacement (mm) & .06 & 1.32 & .66 & .39 \\
\hline
\end{tabular}

Note: St Dev = standard deviation; A-P = anteroposterior 


\section{Figure Captions}

Figure 1. Audio signal (top panel), formant frequencies (second panel), magnet position (third panel), and the acoustic-kinematic continuous correlations (lower panel) for one repetition of the word boy. The operationally defined onset, transition, and offset of the diphthong are indicated in the second panel and all signals are time-aligned. Solid blue lines are indicative of F1/vertical movements; dotted red lines reflect anteroposterior movements. All dependent measures for the study were derived from the diphthong transition segment.

Figure 2. Audio signal (top panel), formant frequencies (second panel), magnet position (third panel), and the acoustic-kinematic continuous correlations (lower panel) for one repetition of the word shout. The operationally defined onset, transition, and offset of the diphthong are indicated in the second panel and all signals are time-aligned. Solid blue lines are indicative of F1/vertical movements; dotted red lines reflect anteroposterior movements. All dependent measures for the study were derived from the diphthong transition segment.

Figure 3. Individual speaker acoustic-kinematic correlations (mean of three tokens, of which each was the mean Pearson $r$ for the diphthong transition) for the four diphthongs, showing vertical data in the left column and anteroposterior in the right.

Figure 4. Kinematic x-y trajectories (left) and F1/F2 formant space histories (right), showing three tokens of the diphthong /av/ for different speakers. The upper panels show how the three repetitions of the diphthong by speaker 7 had consistent lingual movements, but variable formant tracks. The middle panels reveal that speaker 18 had token-to-token variability in the position, but not the pattern of the kinematic traces, 
with only modest differences in the acoustic record. The lower panels show the same diphthong for speaker 4, who had lingual movements that were more variable than the formant histories for the three tokens. 

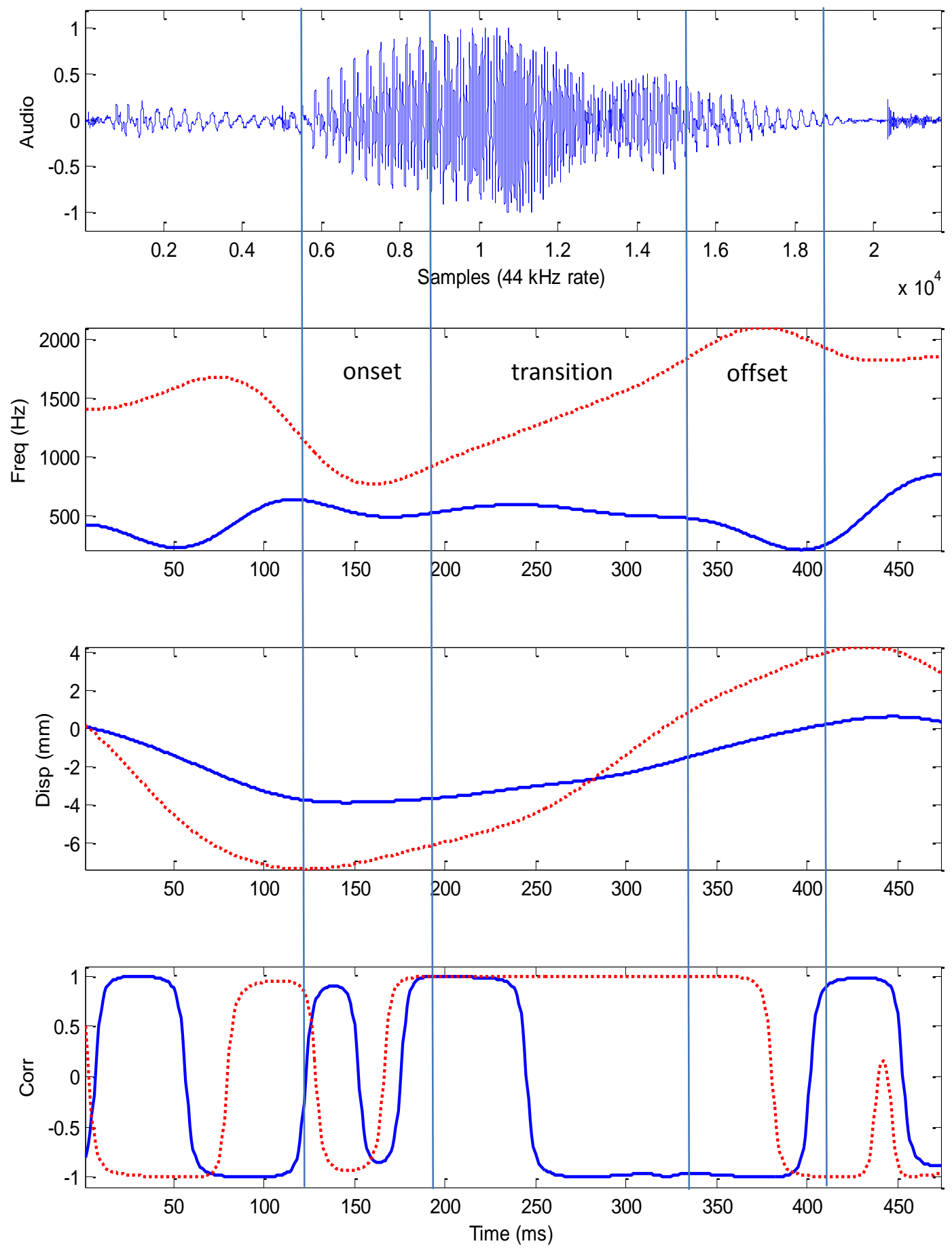


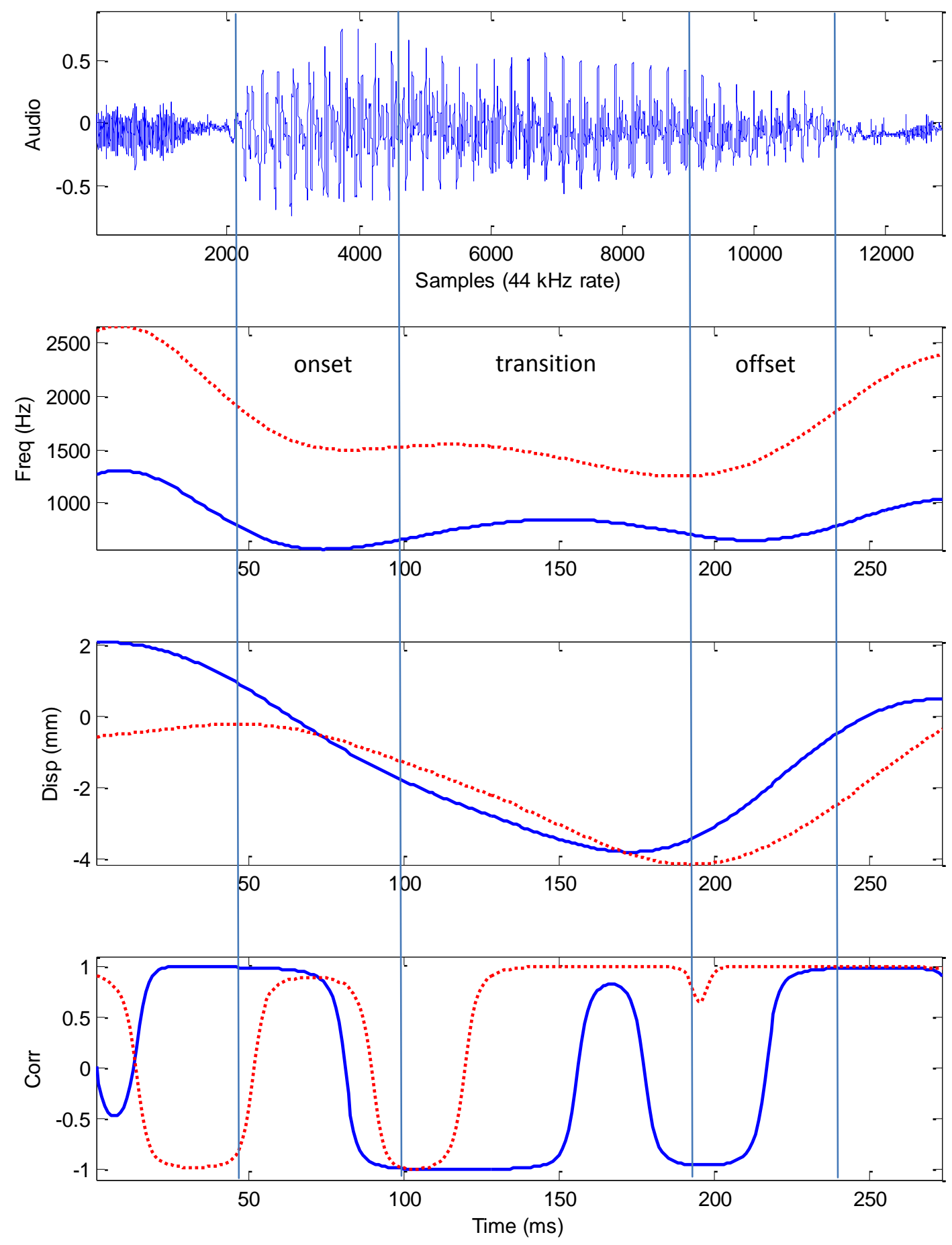


F1 / Vertical
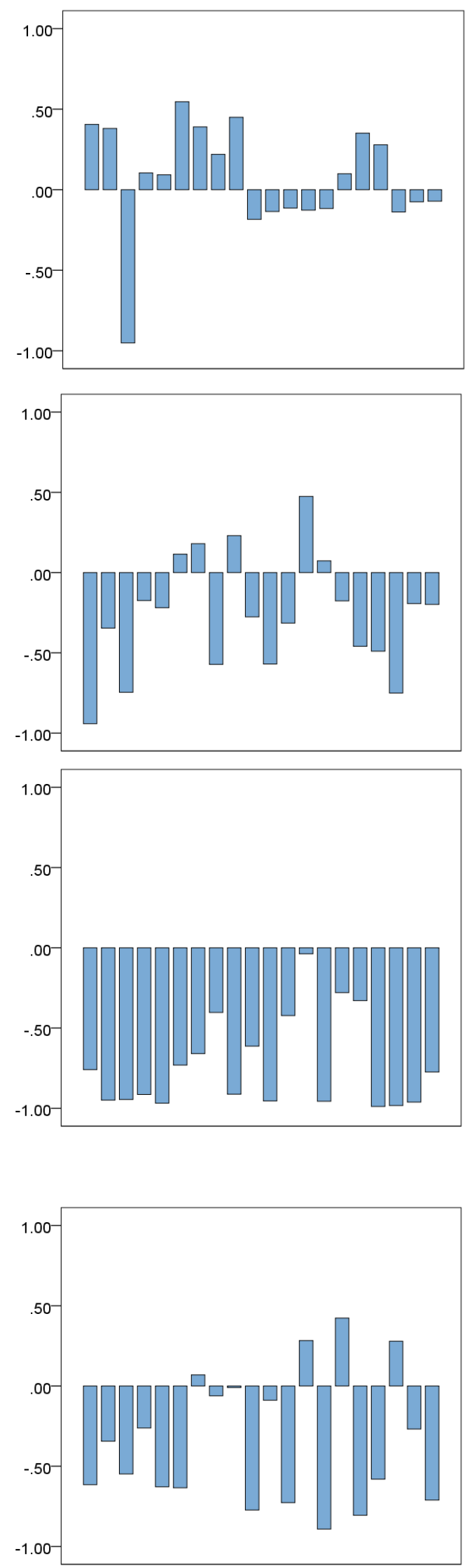

F2 / anteroposterior
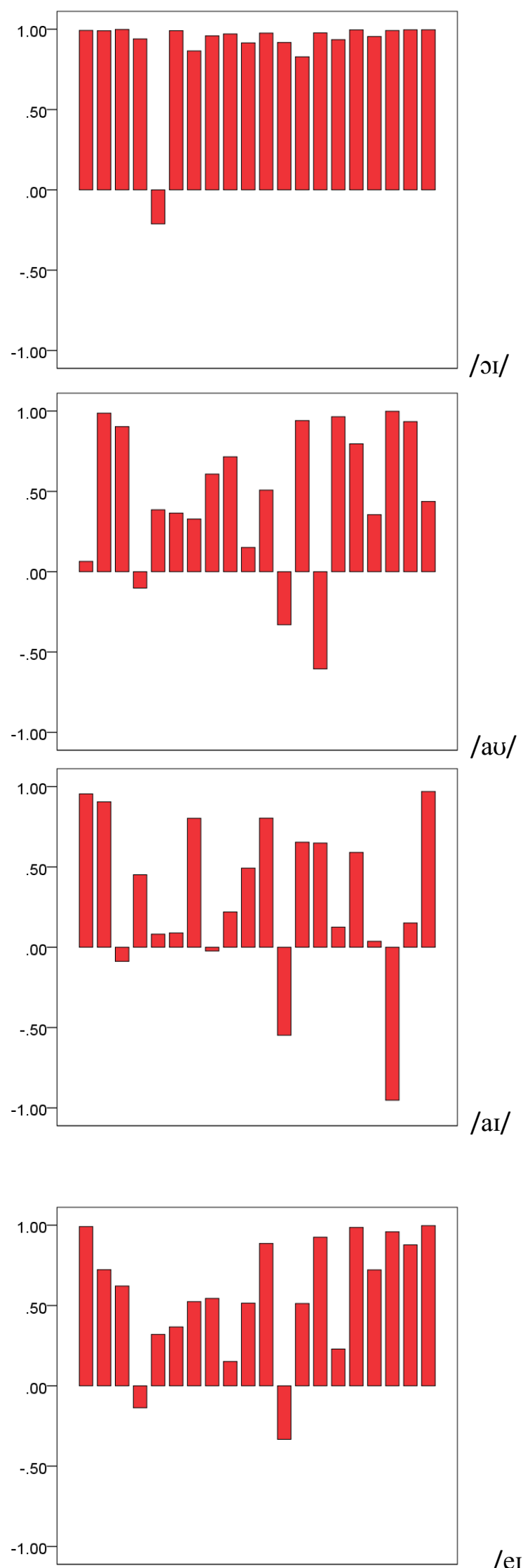

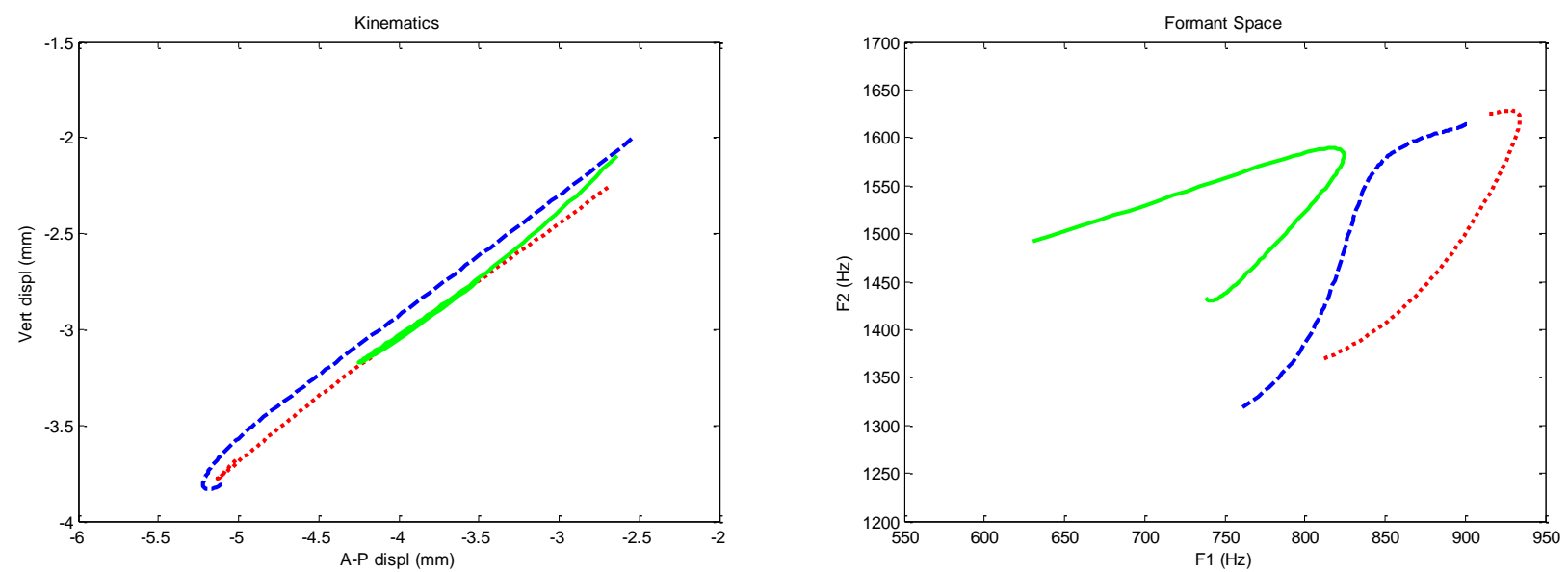

speaker 7
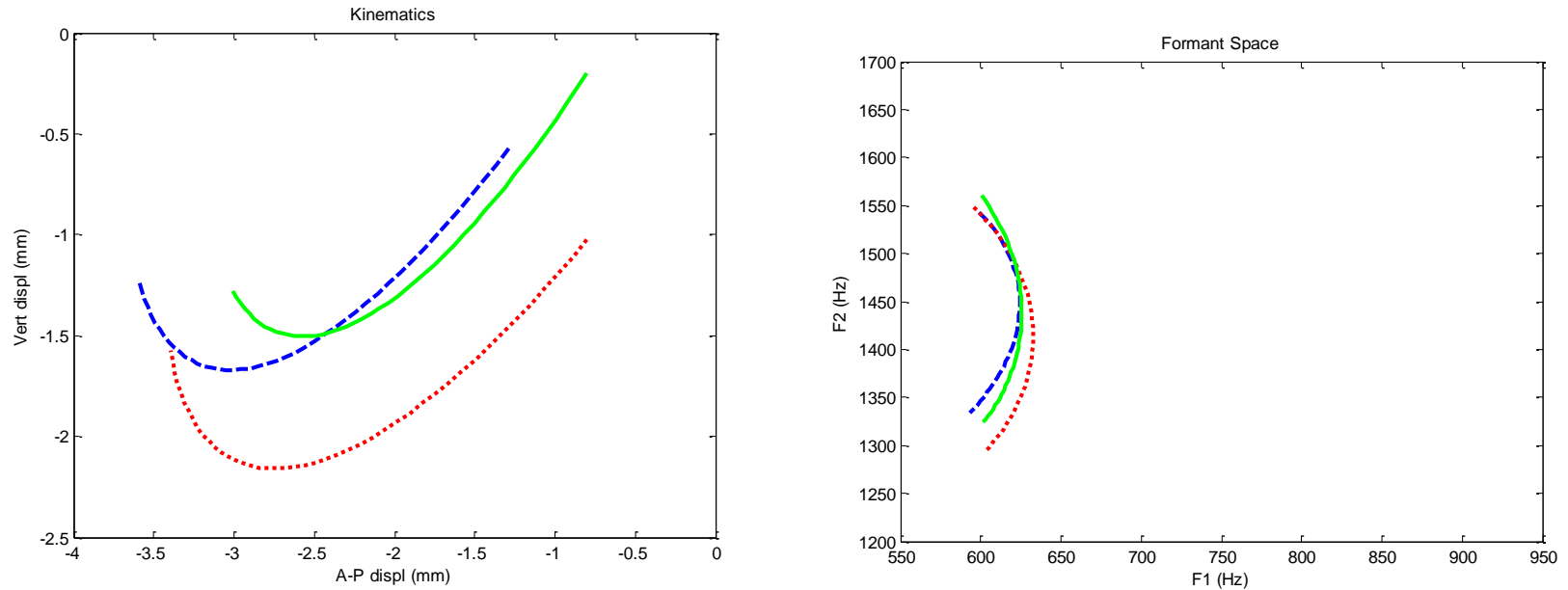

speaker 18
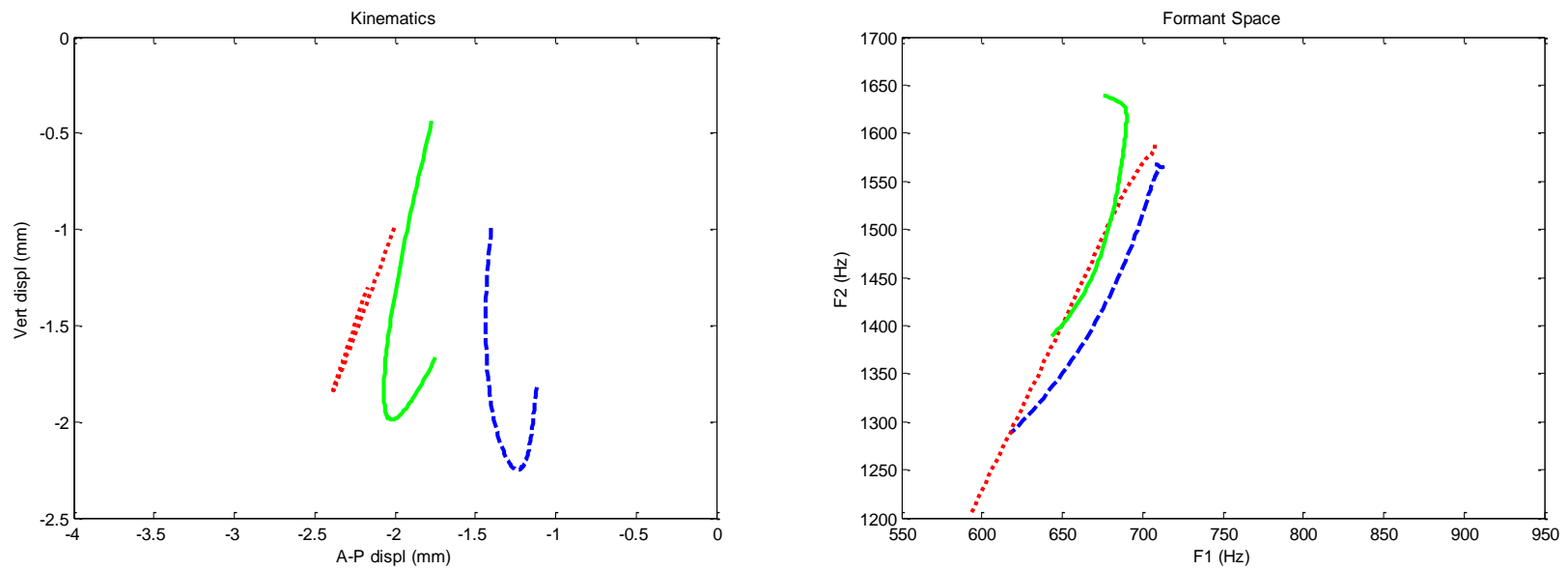

speaker 4 